\title{
Diagnostic value of the Rotterdam-CAMCOG in post-stroke dementia
}

\author{
I de Koning, F van Kooten, P J Koudstaal, D W J Dippel
}

J Neurol Neurosurg Psychiatry 2005;76:263-265. doi: 10.1136/jnnp.2004.03951 1

\begin{abstract}
Background and Objective: Specific screening tests to detect post-stroke dementia are lacking. We recently reported that an adaptation of the Cambridge Cognitive Examination (CAMCOG), the Rotterdam-CAMCOG, had excellent sensitivity and specificity for detecting post-stroke dementia. In this study, we externally validated the diagnostic accuracy of the $\mathrm{R}-\mathrm{CAMCOG}$ in a new, representative cohort of stroke patients.

Methods: The R-CAMCOG and an extensive neuropsychological examination were administered, independently of each other, in 121 patients aged 55 and over with a stroke in the preceding three to nine months. The gold standard diagnosis of dementia was based on the results of the extensive neuropsychological examination, clinical presentation, and information from a close relative, as well as DSM-IV criteria.

Results: Of the 121 patients, 35 had dementia (29\%). The diagnostic accuracy at the pre-specified cut-off point of 33/ 34 was established through receiver operating characteristic (ROC) analyses (sensitivity $66 \%$, specificity $94 \%$ ). At a cut-off point of $36 / 37$ sensitivity would be $83 \%$ and specificity $78 \%$. Conclusion: The R-CAMCOG is a useful screening tool for post-stroke dementia in a clinical setting.
\end{abstract}

$\mathrm{U}$ ntil recently, no specific screening tests were available for the detection of post-stroke dementia. This led to an increased risk of misclassification in screening procedures, as most screening tests are aimed at cortical disturbances, consistent with Alzheimer's disease. We recently reported that the Cambridge Cognitive Examination (CAMCOG) is suitable for screening in post-stroke dementia, ${ }^{1}$ but it has a major drawback, namely the relatively lengthy administration time of approximately 25 minutes. We therefore developed a modified version of the CAMCOG, the Rotterdam-CAMCOG, which is easy to administer, taking approximately 10 minutes. ${ }^{2}$ This is an important aspect of stroke management because of the high caseload: approximately a quarter of all hospitalised stroke patients will have dementia. ${ }^{3-5}$ A preliminary analysis of the diagnostic value of the R-CAMCOG on the dataset used to develop the test suggested that the high sensitivity and specificity were preserved after adaptation. ${ }^{2}$ However, external validation and assessment of reliability in a different series of patients with stroke was necessary to determine the value of the RCAMCOG as a screening instrument for dementia in patients with a recent stroke. In the present study, we prospectively assessed the diagnostic accuracy of the R-CAMCOG in a new representative cohort of 121 patients with stroke.

\section{METHODS}

\section{Patients}

Between October 2000 and July 2002 patients with stroke from various centres in the region (Rotterdam-Rijnmond) were enrolled in the study. The patients had to be 55 years or older and have had a transient ischaemic attack (TIA) with neurological signs on examination, ischaemic stroke, or intracerebral haemorrhage in the preceding three to nine months. The stroke had to be confirmed by a neurologist, and neuroimaging had to be available. Patients were excluded when a reliable assessment of dementia could not be made because of aphasia (that is, a score less than 3 on the Aphasia Severity Rating Scale from the Boston Diagnostic Aphasia Examination), ${ }^{6}$ severe sensory handicaps (for example, deafness or blindness), persistent impairment in consciousness, severe psychiatric symptoms, or insufficient command of the Dutch language. Additional criteria for exclusion were a TIA without neurological signs on examination, concomitant primary cerebral disorder or severe comorbidity with a short life expectancy. Informed consent was obtained from all patients or from close relatives in case of impaired judgement. The local medical ethics committee approved the study.

\section{Procedure}

In each centre, consecutive eligible patients were asked (or their families) to participate in the study. At inclusion, detailed information about cardiovascular risk factors, stroke characteristics, and premorbid status was obtained from the patient's files. Premorbid cognitive functioning was assessed by interviewing a close relative and the score on the Blessed Dementia Scale ${ }^{7}$ to establish prestroke cognitive decline or dementia. Education was categorised into levels from $1=$ less than six years of primary education to $7=$ academic schooling. ${ }^{8}$ Between three and nine months after the onset of stroke two trained research assistants administered either the R-CAMCOG or an extended neuropsychological examination, independently of each other. We used the same neuropsychological test battery as in our previous study. ${ }^{1}$ The research assistant who administered the R-CAMCOG was unaware of the neuropsychological test results, and vice versa. The "gold standard" diagnosis of dementia was based on the results of the extensive neuropsychological examination, clinical presentation, and information from a close relative. When a patient was not able to perform a test due to a motor handicap, we used the results of the non-motor counterpart(s).

In some patients not all tests could be administered due to cognitive disturbances and these patients were only included when a reliable judgement of cognitive functioning could be made. The final judgement of cognitive functioning was made by a diagnostic panel (neurologist and neuropsychologist). DSM-IV criteria were used for the assessment of dementia, ${ }^{9}$ which was further subclassified according to the research criteria of the NINDS-AIREN international workshop, ${ }^{10}$ where possible Alzheimer's disease (AD) and cerebrovascular disease ( $\mathrm{AD}$ and $\mathrm{CVD}$ ) is a potential diagnosis next to probable and possible vascular dementia (VaD).

Abbreviations: CAMCOG, Cambridge Cognitive Examination; RCAMCOG, Rotterdam-CAMCOG; TIA, transient ischaemic attack 


\begin{tabular}{|c|c|c|c|c|}
\hline & $\begin{array}{l}\text { Patients with } \\
\text { dementia } \\
(\mathrm{n}=35)\end{array}$ & $\begin{array}{l}\text { Patients without } \\
\text { dementia } \\
\text { ( } n=86 \text { ) }\end{array}$ & $\begin{array}{l}\text { Total } \\
\text { ( } n=121)\end{array}$ & p value \\
\hline Age (SD) & $74.9(8.8)$ & $68.1(8.3)$ & $70(9.0)$ & $<0.001$ \\
\hline Years of education (SD) & $8.9(4.1)$ & $9.6(3.1)$ & $9.4(3.4)$ & 0.17 \\
\hline \multicolumn{5}{|l|}{ Level of education } \\
\hline Primary school & $17(48 \%)$ & $27(31 \%)$ & $44(37 \%)$ & \\
\hline Vocational education & $10(29 \%)$ & 28 (33\%) & $38(31 \%)$ & \\
\hline Secondary education & $8(23 \%)$ & $31(36 \%)$ & $39(32 \%)$ & 0.18 \\
\hline No. of women & $13(37 \%)$ & $33(38 \%)$ & $46(38 \%)$ & 0.9 \\
\hline \multicolumn{5}{|l|}{ Type of stroke } \\
\hline TIA & $0(0 \%)$ & $25(29 \%)$ & $25(21 \%)$ & \\
\hline Infarction & $27(77 \%)$ & $50(58 \%)$ & $77(63 \%)$ & \\
\hline Intracerebral haemorrhage & $8(23 \%)$ & 11 (13\%) & $19(16 \%)$ & 0.001 \\
\hline \multicolumn{5}{|l|}{ Site of stroke } \\
\hline Infratentorial & $1(3 \%)$ & $9(11 \%)$ & $10(8 \%)$ & \\
\hline Right hemisphere & $23(66 \%)$ & $39(46 \%)$ & $62(52 \%)$ & \\
\hline Leff hemisphere & 11 (31\%) & $36(43 \%)$ & $47(40 \%)$ & 0.11 \\
\hline \multicolumn{5}{|l|}{ Computed tomography (CT) variables } \\
\hline \multicolumn{5}{|l|}{ Main findings $(n=120)$} \\
\hline Normal CT & $3(9 \%)$ & $29(34 \%)$ & $32(26 \%)$ & \\
\hline Recent infarct only & $15(43 \%)$ & $27(32 \%)$ & $42(35 \%)$ & \\
\hline Old infarct only & $4(11 \%)$ & $10(12 \%)$ & $14(12 \%)$ & \\
\hline Single intracerebral haemorrhage & $7(20 \%)$ & $7(8 \%)$ & $14(12 \%)$ & \\
\hline Multiple lesions & $6(17 \%)$ & $12(14 \%)$ & $18(15 \%)$ & 0.05 \\
\hline \multicolumn{5}{|l|}{ Recent infarct type $(n=58)$} \\
\hline Large (end zone and/or large deep) & $17(81 \%)$ & $21(57 \%)$ & $38(66 \%)$ & \\
\hline Lacunar & $4(19 \%)$ & $16(43 \%)$ & $20(34 \%)$ & 0.06 \\
\hline Leukoaraiosis $(n=120)$ & $19(54 \%)$ & $23(27 \%)$ & $42(35 \%)$ & 0.004 \\
\hline Cerebral atrophy $(n=120)$ & $9(26 \%)$ & $14(16 \%)$ & $23(19 \%)$ & 0.24 \\
\hline $\begin{array}{l}\text { R-CAMCOG subscores (SD) } \\
\text { Orientation (maximum }=8 \text { ) }\end{array}$ & $5.5(2.1)$ & $7.6(0.6)$ & $7.0(1.6)$ & $<0.001$ \\
\hline Memory $($ maximum $=25)$ & $14.1(4.5)$ & $20.1(2.7)$ & $18.4(4.3)$ & $<0.001$ \\
\hline Abstraction (maximum $=8$ ) & 4.7 (2.2) & $6.3(1.5)$ & $5.8(1.9)$ & $<0.001$ \\
\hline Perception (maximum $=8$ ) & $4.5(1.3)$ & $6.1(1.3)$ & 5.7 (1.5) & $<0.001$ \\
\hline Total $($ maximum $=49)$ & $28.8(8.2)$ & $40.1(4.1)$ & $36.8(7.6)$ & $<0.001$ \\
\hline
\end{tabular}

\section{Statistics}

The clinical characteristics of the patients with and without dementia were compared using the Student's $t$ test and the $\chi^{2}$ test when appropriate. The diagnostic accuracy (sensitivity and specificity) of the R-CAMCOG was estimated with receiver operating characteristic (ROC) analyses at a prespecified cut-off point of 33/34. ${ }^{2}$ All statistical analyses were carried out with Stata software. ${ }^{11}$

We estimated that a sample size of 150 , with a prevalence of dementia of $20 \%$ would provide sufficiently accurate sensitivity and specificity (standard error of the mean: 0.05 and 0.03 , respectively), however, for logistic reasons, the total number of patients included was lower (121 v 150). Before analysing the data we noted that the frequency of patients with dementia was higher than expected before the study (29\% v 20\%). We therefore assumed that the precision (and standard error) of our estimates would not be affected by the smaller sample size.

Of 130 patients considered eligible by the treating physician, nine had to be excluded: two patients were younger than 55 years, two patients had a history of psychiatric illness, in two (nursing home) patients the diagnosis of stroke could not be confirmed, and in three patients informed consent was withdrawn after initial consent.

The baseline characteristics of the study population are shown in table 1 (mean age 70 years; approximately $40 \%$ women). Patients with dementia were seven years older than the patients without dementia $(p<0.001)$. There was no significant difference in the level of education. None of the patients who had had a TIA had dementia. Six patients had dementia before their most recent stroke ( $\mathrm{AD}, 2 ; \mathrm{VaD}, 4)$. The total score and subtest scores on the R-CAMCOG were significantly lower in the demented group.

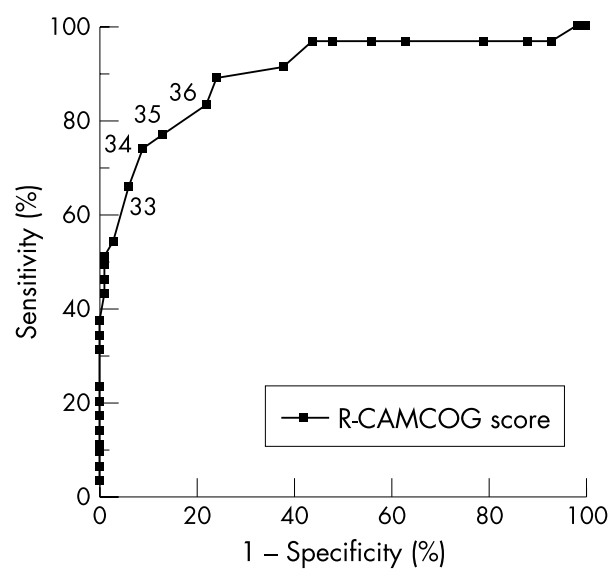

Figure 1 Receiver operating characteristic analysis curve to determine the diagnostic accuracy of R-CAMCOG in a prospective study.

The diagnostic accuracy and test characteristics of the RCAMCOG were determined at the prespecified cut-off value of 33/34, which suggested the highest diagnostic accuracy in a previous study (fig 1). The sensitivity at this cut-off value was $66 \%$ (95\% confidence interval (CI) $50 \%$ to $82 \%$ ) and the specificity was $94 \%$ (95\% CI $89 \%$ to $99 \%$ ), with a positive predictive value of $82 \%$.

\section{DISCUSSION}

In the present study we aimed to confirm the diagnostic accuracy of the R-CAMCOG in a representative stroke population. The findings of this study are different from our previous study in which the R-CAMCOG was developed. 
Not surprisingly, the sensitivity and specificity of the RCAMCOG was higher in the former study since it provided the data used to construct the test. This can be attributed to several types of optimism bias that come into play during the selection and evaluation of predictors. ${ }^{12}$ In fact, this provided the rationale for conducting the present study.

The clinical validity and usefulness of the R-CAMCOG depend on its accuracy and ease of use. In this respect, a limitation of this study was that we did not directly compare the R-CAMCOG with the Mini Mental State Examination (MMSE) or other tests. At the prespecified cut-off point, the sensitivity of the R-CAMCOG was estimated at 66\% (95\% CI $50 \%$ to $82 \%$ ). The MMSE's sensitivity was $69 \%$ (95\% CI 57\% to $81 \%$ ) at the accepted cut-off value of $23 / 24$ in our previous study on vascular factors in dementia in a very similar stroke population. The R-CAMCOG, however, has higher specificity (94\% v 84\%). ${ }^{1}$

High specificity is of great importance, because the diagnostic workup in screen-positive patients is time consuming for the patient and expensive. The moderate sensitivity at the cut-off point of 33/34 may be a disadvantage in the light of the recent development of new and experimental treatments for post-stroke dementia. For these purposes it may be useful to set the cut-off value higher, as is frequently done with screening instruments in other studies. With a cut-off point of $36 / 37$ the sensitivity increases considerably to $83 \%$ (95\% CI $71 \%$ to $95 \%)$, while the specificity is still acceptable $(78 \%$; $95 \%$ CI $70 \%$ to $86 \%$ ). Further testing of R-CAMCOG positive patients would yield a diagnosis of dementia in every second patient, while one in every 20 R-CAMCOG negative patients would have dementia "missed" initially.

For the present study, patients with moderate or severe aphasia were excluded because the R-CAMCOG was not considered as a suitable screening instrument in this subgroup. A screening tool for patients with moderate to severe aphasia would have additional value, but may not be easily realised.

Furthermore, a recent study by Graham et al ${ }^{13}$ showed that executive functioning plays an important role in a particular subgroup of vascular dementia-that is, subcortical vascular dementia. It remains unclear whether the lack of executive items in the R-CAMCOG is a drawback when screening for post-stroke dementia. The clinical picture of patients with stroke as well as restraints due to the direct consequences of a stroke, such as hemiparesis or mild aphasia, may place other demands on a screening instrument.

The impetus for developing a feasible and valid instrument to screen for post-stroke dementia stemmed from the relative incompetence of the existing screening instruments for this purpose. Our study results suggest that the R-CAMCOG may be of limited use as a screening instrument in a large population, but is a useful instrument for screening in poststroke dementia in a clinical setting.

\section{Authors' affiliations}

I de Koning, F van Kooten, P J Koudstaal, D W J Dippel, Department of Neurology, Erasmus MC, Rotterdam, the Netherlands

The study "Screening for dementia with the R-CAMCOG in patients with a recent stroke" is supported by a grant of the College voor Zorgverzekeringen (Dutch Board of Health Insurance).

Competing interests: none declared

Correspondence to: I de Koning, Department of Neurology, Erasmus MC, PO Box 2040, 3000 CA Rotterdam, the Netherlands; i.dekoning@ erasmusmc.nl

Received 17 February 2004

In revised form 10 May 2004

Accepted 17 May 2004

\section{REFERENCES}

1 de Koning I, van Kooten F, Dippel DW, et al. The CAMCOG: a useful screening instrument for dementia in stroke patients. Stroke 1998;29:2080-6. 2 de Koning I, Dippel DW, van Kooten F, et al. A short screening instrument for poststroke dementia: the R-CAMCOG. Stroke 2000;31:1502-8.

3 Tatemichi TK, Desmond DW, Mayeux R, et al. Dementia after stroke: baseline frequency, risks, and clinical features in a hospitalized cohort. Neurology 1992;42: 11 185-93.

4 Pohjasvaara T, Erkinjuntti T, Ylikoski R, et al. Clinical determinants of poststroke dementia. Stroke 1998;29:75-81.

5 Barba R, Martinez-Espinosa S, Rodriguez-Garcia E, et al. Poststroke dementia: clinical features and risk factors. Stroke 2000;31:1494-501.

6 Goodglass H, Kaplan E. The assessment of aphasia and related disorders. Philadelphia: Lea \& Febiger, 1983.

7 Blessed G, Black SE, Butler T, et al. The diagnosis of dementia in the elderly. A comparison of CAMCOG (the cognitive section of CAMDEX), the AGECAT program, DSM-III, the Mini-Mental State Examination and some short rating scales. Br J Psychiatry 1991; 159:193-8.

8 Verhage F. Intelligentie en leeftiid. Assen: Van Gorcum, 1964

9 American Psychiatric Association. Diagnostic and statistical manual of mental disorders. Washington, DC: APA Press, 1994.

10 Roman GC, Tatemichi TK, Erkinjuntti T, et al. Vascular dementia: diagnostic criteria for research studies. Report of the NINDS-AIREN International Workshop. Neurology 1993;43:250-60.

11 Stata Corporation. Stata Statistical software: release 6.0. College Station: Stata Corporation, 1999.

12 Steyerberg EW, Bleeker SE, Moll HA, et al. Internal and external validation of predictive models: a simulation study of bias and precision in small samples. J Clin Epidemiol 2003;56:441-7.

13 Graham NL, Emery T, Hodges JR. Distinctive cognitive profiles in Alzheimer's disease and subcortical vascular dementia. I Neurol Neurosurg Psychiatry 2004;75:61-71. 